\title{
Case Management Services to Meet the Complex Needs of Patients with Multiple Sclerosis in the Community-The Successes and Challenges of a Unique Program from the National Multiple Sclerosis Society
}

\author{
Rosalind Kalb, Kathleen Costello, Linda Guiod \\ National Multiple Sclerosis Society, New York, NY, USA
}

\begin{abstract}
n spite of the therapies currently available to treat people with multiple sclerosis (MS), some individuals continue to experience physical, cognitive, and/or emotional changes over the course of the disease that severely compromise function, independence, safety, and quality of life. In addition, co-morbidities are common in people with MS, further impacting health and well-being, as well as the ability to manage their healthcare needs and cope with everyday life. The National MS Society's Edward M Dowd Personal Advocate Program (which is one component of the National MS Society's MS Navigator ${ }^{\circledR}$ Service) is designed to pick up where traditional neurologic care and Society MS Navigators cannot go-into patients' home environments. This centrally administered program contracts with local professional case managers (most often nurses and social workers) to help stabilize individuals with multiple urgent care needs (for example, in cases of abuse or neglect, or inadequate food, housing or medical care, among others) while identifying and implementing longer-term, sustainable solutions leading to greater independence and quality of life. This article outlines the rationale and goals of the Society's case management services, considers successful and unsuccessful outcomes that have occurred since the program's inception, examines the factors that contribute to success or failure for any given patient, and describes future goals.
\end{abstract}

\section{Keywords}

Multiple sclerosis, case management, National Multiple Sclerosis Society, complex care needs, advanced multiple sclerosis, family, care coordination, housing

Disclosures: Rosalind Kalb, Kathleen Costello, and Linda Guiod have nothing to declare in relation to this article.

Review Process: Double-blind peer review.

Compliance with Ethics: All procedures were followed in accordance with the responsible committee on human experimentation and with the Helsinki Declaration of 1975 and subsequent revisions, and informed consent was received from the patients involved in the case studies.

Authorship: All named authors meet the criteria of the International Committee of Medical Journal Editors for authorship for this manuscript, take responsibility for the integrity of the work as a whole and have given final approval for the version to be published.

Received: January 24, 2019

Accepted: March 12, 2019

Citation: US Neurology. 2019;15(1):27-31

Corresponding Author: Rosalind Kalb,

290 Sabino Road. West Bath, ME 04530,

USA. E: rosalind.kalb@nmss.org

Support: No funding was received in

the publication of this article.
Multiple sclerosis (MS) is a chronic, unpredictable disease for which we do not yet have a cure or fully effective treatment. ${ }^{1}$ The available disease-modifying therapies reduce inflammatory activity and progression of disability for many people. However, some individuals experience physical, cognitive, and/or emotional changes over the disease course that severely compromise function, independence, safety, participation, and quality of life. ${ }^{2}$ While these changes can occur at any time, even as initial symptoms, they tend to accumulate as the disease progresses.

In addition, co-morbidities are more common in MS than in the general population, particularly depression, anxiety, and bipolar disorder, ${ }^{3}$ as well as hypertension, diabetes, and heart disease, ${ }^{4}$ fibromyalgia, irritable bowel syndrome, and chronic lung disease. ${ }^{5}$ Co-morbidities in MS not only compromise a person's overall health, but are also associated with a delay in diagnosis and treatment initiation, ${ }^{6,7}$ an increased risk of hospitalizations, ${ }^{8}$ more rapid disease progression, ${ }^{9}$ shorter lifespan, ${ }^{10}$ and reduced quality of life. ${ }^{11}$ Co-morbidities increase with age in the general population. Given that the MS population as a whole is aging, ${ }^{12}$ more individuals with MS are experiencing co-morbidities that impact their health and well-being, as well as their ability to manage their healthcare, and cope, adapt, and problem-solve in their everyday lives.

These problems are common in MS, but not unique to this disease. People diagnosed with chronic illnesses often require complex hospital- and community-based care from a variety of healthcare providers as well as care and support from spouses/partners and extended family members. ${ }^{13}$ Clinicians who treat patients with chronic, progressive neurologic conditions are aware of the psychosocial challenges many of these individuals experience over time. When patients leave their doctors' offices-assuming they could get there in the first place-they often face physical, emotional, social, and economic barriers that feel insurmountable. However, clinicians generally do not have the training, expertise, or time to assist their patients with physical incapacity, social isolation, economic insolvency, severe mental health or cognitive problems, inaccessible housing or homelessness, or even the inability to manage minimal self-care. 


\section{The role of case management}

The Case Management Society of America defines case management as "a collaborative process of assessment, planning, facilitation, care coordination, evaluation, and advocacy for options and services to meet an individual's and family's comprehensive health needs through communication and available resources to promote quality, cost-effective

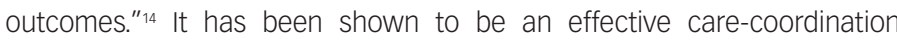
method in the treatment of chronic illness. ${ }^{15,16}$ An additional goal of case management is to help people learn and practice the skills necessary for advocating more effectively for themselves within the healthcare system and the community. In a descriptive qualitative study of older hospitalized adults, the investigators found that older adults value their resilience, which they perceived as being enhanced by the patient-oriented approaches to care that are central to case management. ${ }^{17}$

\section{The role of the National Multiple Sclerosis Society} The National MS Society has the Edward M Dowd Personal Advocate Program in place that is that is designed to pick up where traditional neurologic care cannot go - into patients' homes and daily lives. In this article, we outline the goals of the program, consider successful and unsuccessful outcomes that have occurred since the program's inception, and examine the factors that contribute to success or failure for any given patient.

\section{Background}

In 2016, Edward M Dowd (diagnosed with MS in 1993) gave the National MS Society a $\$ 3$ million-grant to be used specifically for case management services, with the goal of increasing independence and quality of life for people with MS whose health and safety are compromised by limited knowledge and/or the inability to access the care and resources they need. He had experienced first-hand how difficult it can be to identify and utilize services, access quality care, and accomplish even the most basic activities of daily living with MS. He wanted other patients with MS with more limited resources to have the care and support they need to lead full, safe, comfortable, and productive lives.

Case management, as defined at the National MS Society and supported by the Edward M Dowd Personal Advocate Program, is one component of the National MS Society's MS Navigator ${ }^{\circledast}$ Service. MS Navigators respond, on a daily basis, to the complex challenges faced by individuals with MS and their family members. Through a centralized service delivered via phone calls, emails, or online chats, highly trained MS Navigators determine each individual's needs and provide information, referrals for healthcare and local services, support and crisis management, as well as benefits- and employment-counseling.

However, there are limitations to a Navigator's ability to help someone remotely, particularly if the individual (or the care partner) cannot specify the need(s), is feeling overwhelmed, has significant mood or cognitive problems, is at immediate risk, or is simply unable to take the steps necessary to access available benefits or services. What is missing is the opportunity to see people in their home environment.

In prior years, case management was handled by individual National MS Society chapters in different parts of the country, using different eligibility criteria, delivery models, and data collection strategies. To address this variability, the National MS Society determined that a centralized case management strategy was needed across the country.

\section{Program design}

The program is administered centrally, with standardized policies and procedures, data collection, and training requirements for contracted case managers (CMs). The services, which are free for people with MS, are delivered locally by contracted CMs (most often nurses or social workers) who have greater familiarity with state and local resources and easier access to individuals' homes. To ensure that these individuals have an adequate understanding of $\mathrm{MS}$, each $\mathrm{CM}$ is required to complete a threepart educational program prior to receiving any referrals.

The training is accessed through the National MS Society's online learning and development platform in a self-paced format. Part one provides an overview of the disease, with an emphasis on the symptoms of MS that may create challenges in the case management process. Part two covers the processes and procedures that each $\mathrm{CM}$ is expected to use, as well as procedures for protecting confidentiality, and reporting neglect or abuse. Part three reviews the potential impact of MS - financial, emotional, social-on individuals and families, and provides tools and resources to help address those issues.

When an individual's needs go beyond what the MS Navigator is able to provide by telephone or online, that person is eligible for case management. The most common indicators for case management include:

- multiple urgent issues;

- inability for family to cope or provide care;

- isolation or other health or safety risks;

- lack of home care or adequate housing;

- inability to identify needs; and

- potential of admission to a long-term care facility.

At the present time, approximately $2 \%$ of the 50,000 people who contact an MS Navigator in a year are transitioned to the case management program. The CM conducts a home visit that allows for a complete assessment of the situation and makes it possible to address issues already identified by a Navigator, as well as issues that only become apparent during in-person contact and a full standardized assessment.

Case management at the National MS Society is defined as a short-term, intensive service that lasts on average 4-6 months. It is designed to meet immediate needs while identifying and implementing longer-term, sustainable solutions leading to greater safety, independence, and quality of life.

\section{Targeted timeline and process}

The targeted timeline and process for each case is as follows:

- within 3 days of receiving the referral, the contracted CM attempts to schedule a home visit within 5-7 business days;

- within 5 days of the initial visit, the CM completes and submits an Assessment, Consent for Information, and mutually agreed-upon Goal Plan to the Society;

- within 5 days after the case is closed, the CM submits a Case Summary and within 2-4 weeks after the Case Summary is received, a trained volunteer conducts an Outcomes survey by telephone, seeking feedback from the person with MS on the outcomes for each goal, their satisfaction with the program and with the $\mathrm{CM}$.

In reality, each of these steps may take longer, depending on individual circumstances. 
Figure 1: Case management coverage across the United States

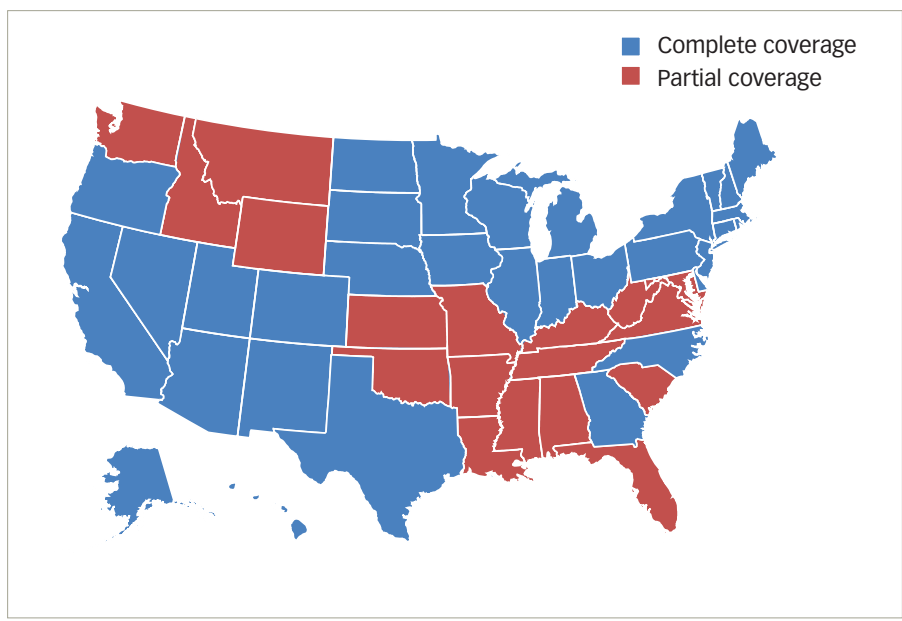

Blue: fully covered states (32 including Washington DC)

Red: partially covered states (18 states)

Source: reused with permission from the National MS Society.

\section{Outcomes to date}

From the rollout of the nationwide case management program in March 2017 through September 2018, 2,391 people with MS have received case management services for an average of 4.7 months. Case management services are available in every state (Figure 1). Every effort is now being made to provide CM services wherever needed, regardless of where the person lives. Data from the follow-up surveys received from 274 program participants indicate that on average $61 \%$ of their goals were met or exceeded, and $20 \%$ of their goals were partially met-an outcome that fell short of the goal of $75 \%$ satisfaction (Figure 2). Over $80 \%$ of participants reported that the service had a positive impact on their lives, and 75\% learned about new resources and felt better able to take action to address future problems. Seventy percent reported feeling that their life had improved after using the service.

The potential reasons for unmet goals have remained consistent since the beginnings of the program. Any or all of the following factors could account for unmet needs in a given situation or a given geographic area:

- insufficient community and government resources in certain areas of the country (no state waivers, lack of accessible housing and transportation, inadequate mental health services);

- person with MS not meeting the eligibility requirements or financial qualifications for existing programs (housing, utility assistance, home modifications);

- long waiting lists for community programs (housing, food stamps, meal delivery);

- lack of client follow-through with recommended resources; and

- person with MS or family members find available solutions unacceptable.

Given that many of these obstacles are systemic, and public policy advocacy at the national and local level is a long-term prospect at best, there are no obvious short-term strategies for addressing unmet goals. In addition, some clients, in spite of the CM's assistance and best efforts, do not, or cannot, follow through on necessary steps. Furthermore, the
Figure 2: Cumulative outcomes in the case management survey



National MS Society supports each individual's right to self-determination, which means that every client has the right to turn down services or solutions that they do not like or do not believe are in their or their family's best interests. The result is that a client's needs may be unmet in a resource-rich area because of personal or family issues that stand in the way, while another client's needs may be met in a resource-poor area because of creativity and determination, or because the missing resources are not needed to address that person's situation.

\section{Setting realistic goals for the future}

As the National MS Society sets goals for 2019 and beyond, it is important to think about what is realistic given the complexity of the problems these individuals and families with MS are facing, the limited resources available in many parts of the country, and the significant physical, emotional, and cognitive symptoms that often stand in people's way. The following case descriptions help to clarify the factors that contribute to success or failure in meeting specific goals.

\section{Jackie-a timeline of events leading to stability and sustainable solutions}

This detailed timeline highlights the complexities of the issues and the time and collaborative effort involved in arriving at sustainable solutions.

\section{October 5, 2016}

Jackie calls an MS Navigator to report:

- the electricity had been shut off;

- Medicaid, food stamps, and home healthcare benefits had been terminated; and

- the client had been homebound for 3 years.

MS Navigator sets several steps in motion:

- a police officer conducts an in-home "welfare check" to determine Jackie's status and calls the paramedics to evaluate her health; and

- a police officer brings food and gets the electricity turned back on for 30 days. 


\section{October 5-November 2, 2016}

The National MS Society's internal case management team:

- prioritizes Jackie's situation as urgent, and sends a CM to her home;

- provides financial assistance through an Energy Outreach Colorado grant to pay utility bills; and

- pays for food in the short term.

The CM conducts a home assessment to identify specific goals:

- reinstate benefits including food stamps and re-enrollment in Home and Community Based Services Program (HCBS; Medicaid waiver for in-home care);

- obtain a lifeline alert for emergency backup;

- obtain meal delivery; and

- address accessibility/safety issues in the home.

November 8, 2016-July 6, 2017

The $\mathrm{CM}$ and Jackie work together to achieve identified goals:

- Medicaid reinstatement after 2 months;

- prescriptions refilled and incontinence supplies ordered;

- food stamps, HCBS services, and Lifeline reinstated;

- neighbor agrees to help with grocery shopping;

- The CM helps Jackie complete application for meal delivery through Project Angelheart:

- The CM helps Jackie complete a successful application to Home Builders Foundation for free home safety/accessibility modifications;

- The CM helps Jackie apply to the state's Low-Income Energy Assistance Program for assistance with utility bill; and

- The CM assists Jackie to obtain free furnace repair from Crisis Intervention Program through Energy Outreach Colorado.

\section{March 21, 2017}

Additional goals added:

- The CM provides Jackie with information about accessing dental and vision coverage with Medicare and Medicaid, which Jackie pursues on her own;

- The CM fills out application for a computer grant so that Jackie can feel more connected to the world;

- The CM assists Jackie with Medicare paperwork and billing questions;

- The CM assists Jackie with the paperwork required for redetermining eligibility and reapplying for public assistance.

\section{July 6, 2017}

Jackie's case is closed following a review of her goals and accomplishments and a reminder about how to access help in the future. It took 9 months to identify and implement the sustainable solutions that Jackie needed to remain safely in her home.

Sustainable solutions for people facing long-term, complex challenges in everyday life involve:

- leveraging thousands of dollars in available community services;

- active participation on the part of people with MS and their family members in the teamwork necessary to complete applications and learn how to self-advocate in the future; and

- a willingness on the part of people with MS and their family members to consider alternatives, make compromises, and remain flexible in their problem-solving efforts.

\section{When goals cannot be met-the path(s) to early case closure \\ BW-50-year-old female}

- B lives with her husband and 11-year-old child.

- The MS Navigator involves a CM to provide B with options for increased safety and independence.

- The CM connects B to a home care agency and The Arc of Montgomery County for respite program subsidy, which was never implemented due to lack of follow-up by the husband.

- B declines participation in adult day programs.

- B does not follow through with inpatient rehabilitation program and subsequent in-home physical and occupational therapy services.

- B and her husband do not follow up on referral to Center for Independent Living for a home modification grant.

- B and her husband decline counseling services.

- B and her husband do not pursue an application for MetroAccess to improve accessibility in their home.

- B's husband declines Meals on Wheels.

The case management file is closed due to lack of follow-up with offered services and poor compliance.

\section{GL-35-year-old female}

- G lives with her boyfriend and child.

- G needs stable, appropriate housing to address her medical, physical, and mental health needs, and to reduce her isolation.

- The highly experienced CM assigned to her case feels unsafe and uncomfortable during the home visit; $\mathrm{G}$ is nonverbal and the boyfriend is behaving oddly.

- G decompensates psychiatrically shortly after the home visit.

The case management file is closed due to severe mental health issues and the case is opened with both Adult Protective Services and the Administration for Children's Services.

JW-48-year-old female

- $\mathrm{J}$ is a single parent of a teenage daughter who provides hands-on care for her mother.

- $J$ is confined to her bed; she has a suprapubic catheter and pressure sores, which are managed by a visiting nurse.

- I needs case management services to coordinate options for continuing care that would relieve her daughter of caregiving responsibilities and identify options for her daughter if J enters a long-term care facility.

- J's cognitive and mobility challenges make it difficult for her to complete paperwork and follow up with resources.

- $\mathrm{J}$ is unwilling to move to an assisted living facility without her daughter.

- A CM arranges short-term funding through the National MS Society and other agencies for home care services; long-term services through the state are not available because J does not meet income requirements.

- J declines offered support or connection opportunities through the National MS Society.

The case management file is closed due to J's ineligibility for ongoing care through the county or state. 
These cases illustrate that goals may not be fully or totally met for a variety of reasons, including lack of available resources in the community, lack of participation or follow-through on the part of the person with MS, and complex cognitive or emotional issues that make the efforts impossible. More work is needed to determine which, if any, of these factors are modifiable, and what strategies are needed for achieving a higher success rate.

\section{Priorities for 2019}

Significant growth has occurred in the national case management program since it began in 2017. Plans are underway in two major areas for the coming year-more systematic and targeted data collection, and case management by telephone in areas that are sparsely populated.

\section{More systematic and targeted data collection}

The early emphasis of the Edward M Dowd Personal Advocate Program was to implement the nation-wide service as quickly as possible. With the program well underway in most urban and many rural areas across the country, it is time to gather more specific information about what is working, what is not working as well, and why not.

\section{Case management by telephone in sparsely populated areas}

The focus of the case management program to date has been on geographic areas with significant numbers of people with MS who have a need for these services. The National MS Society's ultimate goal is to be able to respond to all case management needs, wherever the person may be located. While the program has been able to respond to most case management requests, occasional requests come from people with very extensive needs who live in very sparsely populated areas with no available case management services. The MS Navigators are able to provide a great deal of help and support, but on-the-ground professionals with local knowledge and connections can have a greater impact. In such situations, a local CM may be better able to guide the person through the steps needed to access resources, even if the service is provided locally by phone. This option is offered only for a very small number of people, and only after all other options for in-person case management have been explored.

The 2017 hurricane in Florida offered a unique opportunity to pilot case management services by telephone. People with MS in hard-hit areas were calling the National MS Society for assistance at the same time that many of the contracted case management providers were displaced from their work places or unable to travel to hard-hit areas. However, these providers were knowledgeable about local resources and willing to provide guidance to the National MS Society's clients by telephone, making the pilot program possible. The experiences in Florida, as well as some other more remote areas around the country, have demonstrated that case management services delivered by telephone are partially effective. However, there appears to be a higher attrition rate for telephonic case management. More study is needed to understand the differential rates of attrition and identify strategies to overcome them. In the meantime, efforts are being made to engage CMs in more sparsely populated areas and identify contracted CMs who are willing to expand their coverage areas.

\section{Networking and learning opportunities for contracted case managers}

In spite of the required training program for contracted CMs, some CMs have provided feedback to the National MS Society about the challenges of working with these individuals with MS and their families. Some of these CMs are experienced and comfortable with providing case management services for the aging population, many of whom have family members involved who can participate as needed. They are much less familiar with the complex physical, emotional, cognitive, and social aspects of MS that can interfere with a person's ability to participate fully in the collaborative case management process, particularly when no family members are available to assist with the case management process. The plan in 2019 is to offer group web- or phone-based training that will address some of these challenges with additional information and interactive case-study discussions.

\section{Summary}

Many individuals with MS experience physical, cognitive and/or emotional changes over the course of the disease, as well as comorbid health conditions, which severely compromise function, independence, safety, and quality of life. The National MS Society's Edward M Dowd Personal Advocate Program (which is one component of the Society's MS Navigator ${ }^{\circledR}$ Service) provides case management services through contracted professional case managers across the country. Case management-which is defined at the National MS Society as a short-term, intensive service lasting an average of 4-6 months - is indicated when an individual's needs go beyond what an MS Navigator can provide remotely. It is designed to meet immediate, urgent needs (for example, lack of food, accessible housing, or medical care; and potential nursing home placement) while identifying and implementing longer-term, sustainable solutions leading to greater safety, independence and quality of life. Sustainable solutions for people facing long-term, complex challenges in everyday life involve:

- leveraging thousands of dollars in available community services;

- active participation on the part of people with MS and their family members in the teamwork necessary to complete applications and learn how to self-advocate in the future; and

- a willingness on the part of people with MS and their family members to consider alternatives and remain flexible in their problem-solving efforts.

While the National MS Society's experience highlights the impact of resource disparity in different parts of the country, it also demonstrates that outcomes, both positive and negative, reflect an interaction of all three of these factors. $\square$
1. Multiple Sclerosis Coalition. The Use of Disease-Modifying Therapies in Multiple Sclerosis: Principles and Current Evidence. 2018. Available at: www.nationalmssociety.org/ getmedia/5ca284d3-fc7c-4ba5-b005-ab537d495c3c/DMT Consensus_MS_Coalition_color (accessed January 17, 2019).

2. Kalb R, Beier M, Benedict RH, et al. Recommendations for cognitive screening and management in multiple sclerosis care. Mult Scler. 2018;24:1665-80.

3. Marrie RA, Reingold S, Cohen J, et al. The incidence and prevalence of psychiatric disorders in multiple sclerosis: a systematic review. Mult Scler. 2015;21:305-17.

4. Marrie RA, Fisk J, Tremlett $\mathrm{H}$, et al. Differing trends in the incidence of vascular comorbidity in MS and the general population. Neurol Clin Pract. 2016;6:120-8.

5. Marrie RA, Reider N, Stuve O, et al. The incidence and prevalence of comorbid gastrointestinal, musculoskeletal, ocular, pulmonary, and renal disorders in multiple sclerosis: a systematic review.
Mult Scler. 2015;21:332-41.

6. Marrie RA, Horwitz R, Cutter G, et al. Comorbidity delays diagnosis and increases disability at diagnosis in MS. Neurology. 2009;72:117-24.

7. Zhang T, Tremlett H, Leung S, et al. Examining comorbidities on disease-modifying therapy use in multiple sclerosis. Neurology. 2016;86:1287-95.

8. Marrie RA, Elliott L, Marriott J, et al. Comorbidity increases the risk of hospitalizations in multiple sclerosis. Neurology. 2015;84:350-8.

9. Marrie RA, Rudick R, Horwitz R, et al. Vascular comorbidity is associated with more rapid disability progress in multiple sclerosis. Neurology. 2010;74:1041-7.

10. Marrie RA, Elliott L, Marriott J, et al. Effect of comorbidity on mortality in multiple sclerosis. Neurology. 2015;85:240-7.

11. Berrigan LI, Fisk JD, Patten SB, et al. Health-related quality of life in multiple sclerosis: direct and indirect effects of comorbidity. Neurology. 2016;86:1417-24.

12. Sanai SA, Saini V, Benedict RH, et al. Aging and multiple sclerosis.
Mult Scler. 2016;22:717-25

13. Joo JY, Liu MF. Experiences of case management which chronic illnesses: a qualitative systematic review. Int Nurs Rev. 2018;65:102-13.

14. Case Management Society of America. Standards of Practice for Case Management. 2016. Available at: http://solutions.cmsa.org/ acton/media/10442/standards-of-practice-for-case-management (accessed January 24, 2019).

15. Joo JY, Liu, MF. Case management effectiveness in reducing hospital use; a systematic review. Int Nurs Rev. 2017;64:296-308.

16. Stokes J, Panagioti M, Alam R, et al. Effectiveness of case management for 'at risk' patients in primary care: a systematic review and meta-analysis. PLoS One. 2015;10:e0132340.

17. Hassani P, Izadi-Avanji FS, Rakshan M, Majd, HA. A phenomenological study on resilience of the elderly suffering from chronic disease: a qualitative study. Psychol Res Behav Manag. 2017;10:59-67. 